\title{
La medición de la salud en el mercado laboral europeo:
}

una perspectiva de género

\author{
Sara Pinillos Franco' \\ Universidad de Valladolid
}

\section{Artículo científico}

Material original autorizado para su primera publicación en el Journal de Ciencias Sociales, Revista Académica de la Facultad de Ciencias Sociales de la Universidad de Palermo.

\section{Recepción: 11-10-2017}

\section{Aceptación: 14-03-2018}

Resumen: Este trabajo de investigación tiene como objetivo comprobar si la salud de los trabajadores es mejor que la de las personas en situación de desempleo en el contexto de la Unión Europea y contrastar la existencia de diferencias de género entre ambos colectivos. Para ello, se han construido dos Indicadores Sintéticos de Salud, uno para empleados y otro para desempleados, con la metodología de la medida de distancia $P_{2}$. La estructura de ambos indicadores refleja que la salud mental y las emociones son importantes a la hora de explicar la salud de los individuos. Tras la obtención de los valores medios de ambos Indicadores Sintéticos de Salud, hemos comprobado la existencia de un patrón territorial caracterizado por un dualismo entre los países del norte, centro y sur de Europa, donde la salud de los empleados y desempleados es mayor, y los países de la Europa del este, donde la salud de ambos colectivos es más pobre, aunque con especial énfasis entre los desempleados. Al analizar las diferencias entre empleados y desempleados en función de su sexo, no se encuentra un patrón claro entre los países, en cambio, al calcular las diferencias entre hombres y mujeres en función de su situación laboral, se puede comprobar que en 18 de los 28 países analizados la salud de las mujeres es mejor que la de los hombres, especialmente si están empleadas. Por lo tanto, la situación laboral afecta a la salud de los hombres y las mujeres de manera diferenciada en función de su país de residencia, por lo que las políticas en materia laboral que desarrollan cada uno de los países juegan un papel importante para mitigar las desigualdades de género en salud.

Palabras clave: mercado laboral; indicador sintético; salud; género.

\footnotetext{
${ }^{1}$ Estudiante de doctorado en Economía dentro del departamento de Fundamentos del Análisis Económico de la Universidad de Valladolid.Correo electrónico: sara.pinillos@uva.es
} 


\section{Measuring health in the European labor market: a gender perspective}

Abstract: This study aims to corroborate whether workers' health is better than the unemployed individuals' health within the European Union framework and to determine the existence of gender disparities between both groups. To achieve this, two Synthetic Health Indicators were created, one for the employed and another one for the unemployed, with the $\mathrm{P}_{2}$ distance method. The structure of both indicators reflects that mental health and emotions are important to explain individuals' health. After obtaining the average values of both Synthetic Health Indicators, we proved the existence of a territorial pattern characterized by a dualism between Nothern, Central and Southern countries, where the employed and unemployed's health is better, and Eastern countries, where both groups' health is poorer, although mostly among the unemployed. When analyzing differences between the employed and the unemployed depending on their sex, a clear pattern across countries is not found, however when calculating men and women differences according to their labor situation, in 18 out of 28 examined countries, women's health is better than men's, in particular, if they are employed. In this vein, labor situation affects men and women differently according to their country of residence, therefore, the labor market policies applied by each country play an essential role to mitigate the gender health inequalities.

Keywords: labor market; synthetic indicator; health; gender.

\section{Introducción}

Según la Organización Mundial de la Salud (OMS), aquellas personas que cuentan con un trabajo remunerado tienen mayor nivel de autoestima y reconocimiento social, lo que repercute positivamente en su salud. Además, si este trabajo es de mayor cualificación y mejor remunerado, su estado de salud autopercibido es mayor, presentando menos trastornos crónicos y mejores indicadores de salud mental (OMS, 2016). El objetivo de este trabajo es doble: por un lado, contrastar si se cumple la hipótesis de que el trabajo incide positivamente en la salud de los trabajadores y trabajadoras y, por otro, comprobar la existencia de diferencias de género en dicha relación en el contexto de la Unión Europea.

En función de las condiciones laborales, en cierta manera condicionadas por los mercados de trabajo, los efectos sobre la salud van a tener diferente signo. Por ejemplo, aquellas personas que cuentan con un trabajo no estándar, disfrutan de peores condiciones laborales (menor remuneración, alto grado de inseguridad, etc.), lo que está asociado a un mayor riesgo de reportar una salud pobre (Kawachi, 2008) y padecer más síntomas depresivos (Quesnel-Vallée, DeHaney y Ciampi, 2010). Por lo tanto, la inseguridad laboral tiene un impacto negativo en la salud de los individuos, aunque con especial énfasis entre las 
mujeres (Rugulies et al., 2008) y las personas desempleadas de larga duración (Böckerman y Ilmakunnas, 2009 y Paul y Moser, 2009).

Desde una óptica de género, cobra una vital importancia la doble dimensión del concepto de trabajo: el reproductivo o no remunerado (cuidado y crianza de hijos, trabajos domésticos, etc.) y el remunerado o productivo. Además, en la mayoría de las sociedades existe una división sexual del trabajo que no resulta equitativa entre hombres y mujeres (Menéndez et al., 2007), lo que provoca mayores niveles de estrés y problemas de salud para las mujeres, puesto que el trabajo reproductivo recae principalmente sobre ellas (Artazcoz et al., 2014).

La balanza de los efectos positivos y negativos del trabajo sobre la salud varía en función del tipo de trabajo, de sus condiciones y de la combinación con los roles productivos y reproductivos. En este contexto, se plantea un trabajo de investigación centrado en abordar las desigualdades de género en salud existentes en el mercado laboral de la Unión Europea de los 28 a través de la estimación de Indicadores Sintéticos de Salud.

\section{Método}

Para aproximarnos a la medición de la salud en el mercado laboral europeo, hemos estimado dos Indicadores Sintéticos de Salud (ISS), uno para personas empleadas y otro para personas desempleadas, a través de la metodología de la medida de distancia $P_{2}$. Esta técnica permite incluir información de diversos indicadores parciales independientemente de su heterogeneidad, pudiendo así abordar el carácter multidimensional de la salud. Además, ofrece la posibilidad de realizar comparaciones interespaciales entre los 28 países que conforman la UE a partir de la información proporcionada por los individuos. Tradicionalmente, la medida de distancia $\mathrm{P}_{2}$ se ha utilizado con unidades espaciales, por lo que una de las novedades de este trabajo de investigación es emplear dicha metodología con unidades a nivel individual. En cuanto al procedimiento de estimación de la $\mathrm{DP}_{2}$ (Somarriba y Pena, 2009 y Somarriba y Zarzosa, 2016), se expresa de la siguiente forma:

$$
\mathrm{DP}_{2}=\sum_{\mathrm{i}=1}^{\mathrm{n}}\left\{\left(\mathrm{d}_{\mathrm{i}} / \sigma_{\mathrm{i}}\right)\left(1-\mathrm{R}_{\mathrm{i}, \mathrm{i}}^{2}, \ldots, 1\right)\right\} \text { con } \mathrm{R}_{1}^{2}=0
$$

Sea $X=\left\{\mathrm{x}_{\mathrm{ij}}\right\}$ la matriz de datos correspondientes a las $\mathrm{m}$ unidades (individuos) (filas) $y$ los $n$ indicadores (columnas) y sea $x_{i j}$ el estado en que se encuentra el componente i-esimo en el individuo $\mathrm{j}$.

Donde $d_{i}=\left|x_{j i}-x_{* i}\right|$ y sean: $\sigma_{i}$ la desviación típica de la variable $i, R_{i, i}^{2} 1, \ldots, 1$ el Coeficiente de Determinación en la regresión de $X_{i}$ sobre $X_{i-1}, X_{i-2, \ldots,} X_{1}, y\left(1-R_{i, i}^{2} 1, \ldots, 1\right)$ actúa a modo de factor corrector eliminando la información duplicada. Se toma como base de referencia 
$\mathrm{X}_{\star}=\left(\mathrm{X}_{*_{1}}, \mathrm{X}_{2}, \ldots, \mathrm{X}_{\mathrm{n}}\right)$, un individuo teórico que alcanza los peores valores de las variables objeto de estudio y cuyo valor del indicador sintético sería cero.

El indicador $\mathrm{DP}_{2}$ devuelve las distancias de cada individuo a ese individuo teórico de referencia, por ello, un valor más elevado de la medida de distancia indica que el individuo está más alejado de ese valor mínimo de salud.

La $\mathrm{DP}_{2}$ cumple todas las propiedades exigibles a un buen indicador de distancia y permite, además, comparaciones intertemporales e interespaciales (Zarzosa Espina, 1996).

\section{Resultados}

Para la elaboración de los ISS se han utilizado los datos que proporciona la Encuesta Europea de Condiciones de Vida (EQLS) del año 2012. Aunque esta encuesta no incluye información relativa a la existencia de enfermedades específicas que puedan padecer los individuos o el número de visitas que una persona realiza al médico de cabecera o al especialista, proporciona información útil sobre la situación socioeconómica de los individuos y otros aspectos que pueden influir en la salud de las personas, tales como la situación del país o el entorno en el que desarrollan sus vidas o, como es el caso que nos ocupa, la situación laboral.

La decisión de crear Indicadores Sintéticos de Salud estriba en la naturaleza multidimensional de este concepto (OMS, 2011), lo que implica la inclusión de diferentes dimensiones para su correcta medición. En el Cuadro 1 aparecen los diferentes indicadores parciales que se han tenido en cuenta para la elaboración de los ISS así como los valores que toman cada uno de ellos. La mayoría de indicadores parciales que se han empleado para la construcción de los ISS son de carácter subjetivo, a excepción del índice de salud mental, la esperanza de vida, la esperanza de vida saludable y la mortalidad infantil. Aunque un ISS idóneo debería incluir también aspectos más objetivos de la salud tales como la presencia de enfermedades o indicadores objetivos sobre la calidad de los servicios sanitarios, no se han considerado debido a la ausencia de los mismos en la base de datos empleada. No obstante, los ISS obtenidos nos permiten aproximarnos al concepto de salud de una manera multidimensional y ofrecer una nueva visión de su composición a lo largo de la Unión Europea para los hombres y las mujeres.

Cuadro 1. Indicadores simples incluidos en el Indicador Sintético de Salud

a. Esta información, proporcionada por la EQLS, ha sido obtenida a partir de la base de datos del Eurostat. 


\begin{tabular}{|c|c|c|c|}
\hline Nivel micro & Valores & Nivel meso & Valores \\
\hline Estado de salud (Q42) & $\begin{array}{c}\text { De } 1 \text { (muy buena) a } 5 \\
\text { (muy mala) }\end{array}$ & $\begin{array}{c}\text { Calidad de los servicios } \\
\text { sanitarios (Q53a) }\end{array}$ & $\begin{array}{l}\text { De } 1 \text { a } 10, \text { siendo } 1 \text { una } \\
\text { calidad muy pobre y } 100 \\
\text { una calidad muy alta. }\end{array}$ \\
\hline $\begin{array}{l}\text { Satisfacción con la salud } \\
\qquad(\mathrm{Q} 40 \mathrm{f})\end{array}$ & $\begin{array}{c}\text { De } 1 \text { (muy } \\
\text { insatisfecho) a } 10 \\
\text { (muy satisfecho) }\end{array}$ & & \\
\hline Sentirse alegre (Q45a) & \multirow{6}{*}{$\begin{array}{c}\text { De } 1 \text { (todo el tiempo) } \\
\text { a } 6 \text { (en ningún } \\
\text { momento) }\end{array}$} & Nivel macro $^{a}$ & \\
\hline Sentirse relajado (Q45b) & & Esperanza de vida & De 68.10 a 85.70 \\
\hline Sentirse vigoroso (Q45c) & & $\begin{array}{l}\text { Esperanza de vida } \\
\text { saludable }\end{array}$ & De 52.10 a 71.10 \\
\hline $\begin{array}{l}\text { Sentirse optimista } \\
\text { (Q45e) }\end{array}$ & & Mortalidad infantil & De 2.10 a 9.40 \\
\hline $\begin{array}{c}\text { Sentirse estresado } \\
\text { (Q46a) }\end{array}$ & & & \\
\hline Sentirse solo (Q46b) & & & \\
\hline $\begin{array}{c}\text { Índice de Exclusión } \\
\text { Social } \\
\text { (Q29e, Q29f, Q29g, } \\
\text { Q29h) }\end{array}$ & $\begin{array}{l}\text { De } 1 \text { a } 5 \text {, siendo } 1 \text { un } \\
\text { mayor nivel de } \\
\text { exclusión social y } 5 \text { un } \\
\text { menor nivel. }\end{array}$ & & \\
\hline $\begin{array}{l}\text { Índice de Salud Mental } \\
\text { (medido a través de la } \\
\text { escala WHO-5) }\end{array}$ & $\begin{array}{l}\text { De } 0 \text { a } 100, \text { siendo } 0 \\
\text { una salud mental muy } \\
\text { pobre y } 100 \text { la mejor } \\
\text { salud mental posible. }\end{array}$ & & \\
\hline
\end{tabular}

Los indicadores parciales seleccionados se han clasificado en tres niveles de salud: micro, meso y macro. La inclusión de estas tres dimensiones permite no solo abordar la multidimensionalidad del concepto, sino también la situación real de los individuos cuando se mide su salud en el momento de la encuesta.

En primer lugar, se ha incluido información a nivel micro, es decir, información que proporcionan los propios individuos. Se ha considerado el estado de salud y la satisfacción de los individuos con su salud, además de ciertas sensaciones acerca de su vida que abarcan aspectos como, por ejemplo, la vitalidad y el rol emocional que afectan a la salud (Michalos, 2003). Además, en este nivel se ha tenido en cuenta la salud mental y los problemas sociales de las personas medidos a través de un Indicador de Exclusión Social, en la línea de trabajos como Chen (1975).

También se ha incorporado información a nivel meso, nivel intermedio entre el nivel micro y macro, a través de la calidad que perciben los individuos sobre los servicios sanitarios de los que disponen. Klomp y Haan (2010) afirman que este nivel permite captar la capacidad 
de los servicios sanitarios que, consecuentemente, afecta al tratamiento de la salud de los individuos.

Finalmente, se ha considerado el nivel macro de salud, que se relaciona con la duración de la vida, puesto que "es muy común aproximarse al estado de salud de la población de un país a través de su esperanza de vida o su tasa de mortalidad" (Klomp y Haan, 2010). En este nivel se ha tenido en cuenta la tasa de mortalidad infantil, la esperanza de vida de los individuos, así como su esperanza de vida saludable.

Tras la selección de los indicadores parciales y la eliminación de los valores perdidos para analizar casos completos, la muestra final estaba compuesta por un total de 17,761 individuos, de los cuales 15,199 estaban empleados y 2,562 en situación de desempleo. En el Cuadro 2 se muestran los valores medios de cada uno de los indicadores utilizados para la construcción de los ISS. Se puede comprobar que los hombres, tanto empleados como desempleados, declaran estar más satisfechos con su salud y con la calidad de los servicios sanitarios que las mujeres en su misma situación laboral. No obstante, también apreciamos que se sienten más estresados y cuentan con una esperanza de vida y una esperanza de vida saludable más baja. Por el contrario, las mujeres se sienten más alegres, relajadas, vigorosas y optimistas, sin embargo, perciben su salud de manera más pobre y presentan una menor puntuación en su índice de salud mental que los hombres.

Cuadro 2. Valores medios de los indicadores simples por situación laboral

Nota: los datos corresponden a la media aritmética de cada uno de los indicadores parciales incluidos en los Indicadores Sintéticos.

\begin{tabular}{|l|r|r|r|r|}
\hline & \multicolumn{2}{|c|}{ Empleados } & \multicolumn{2}{c|}{ Desempleados } \\
\hline & Hombres & Mujeres & Hombres & Mujeres \\
\hline Satisfacción con la salud & 7.42 & 7.17 & 7.40 & 7.25 \\
\hline Estado de salud & 2.23 & 2.36 & 2.26 & 2.35 \\
\hline Sentirse alegre & 2.65 & 2.79 & 2.68 & 2.77 \\
\hline Sentirse relajado & 2.75 & 3.03 & 2.80 & 2.97 \\
\hline Sentirse vigoroso & 2.83 & 2.99 & 2.76 & 2.92 \\
\hline Sentirse optimista & 2.81 & 2.94 & 2.77 & 2.89 \\
\hline Sentirse estresado & 4.59 & 4.35 & 4.57 & 4.45 \\
\hline Sentirse solo & 5.22 & 4.99 & 5.20 & 5.02 \\
\hline Calidad de los servicios sanitarios & 6.25 & 6.11 & 6.38 & 6.07 \\
\hline Índice de Exclusión Social & 2.19 & 2.24 & 2.22 & 2.25 \\
\hline Índice de Salud Mental & 63.96 & 60.10 & 64.15 & 61.21 \\
\hline Mortalidad Infantil & 4.08 & 4.07 & 4.21 & 4.19 \\
\hline Esperanza de vida & 76.58 & 82.54 & 76.53 & 82.39 \\
\hline
\end{tabular}




\subsection{Estructura de los indicadores sintéticos de salud por situación laboral}

En el Cuadro 3 se recoge la estructura de los Indicadores Sintéticos de Salud diferenciando entre aquellas personas empleadas y las que se hallan en situación de desempleo. Las variables aparecen ordenadas acorde al grado de correlación en valor absoluto $(|r|)$ de cada una de ellas con el Indicador Sintético de Salud. Además, se incluye el factor corrector $\left(1-R^{2}\right)$, que indica el porcentaje de información nueva que incorpora cada variable al Indicador Sintético de Salud.

Cuadro 3. Estructura de los ISS por situación laboral

\begin{tabular}{|c|c|c|c|c|c|}
\hline Empleados & $1-R^{2}$ & $|\mathbf{r}|$ & Desempleados & $1-R^{2}$ & $|\mathbf{r}|$ \\
\hline Índice de Salud Mental & 1.00 & 0.76 & Índice de Salud Mental & 1.00 & 0.75 \\
\hline Sentirse alegre & 0.32 & 0.69 & Sentirse alegre & 0.33 & 0.69 \\
\hline Sentirse deprimido & 0.74 & 0.65 & Sentirse deprimido & 0.71 & 0.67 \\
\hline Sentirse relajado & 0.33 & 0.63 & Sentirse relajado & 0.33 & 0.63 \\
\hline Sentirse vigoroso & 0.29 & 0.61 & Sentirse optimista & 0.32 & 0.61 \\
\hline $\begin{array}{c}\text { Satisfacción con la } \\
\text { salud }\end{array}$ & 0.75 & 0.60 & Satisfacción con la salud & 0.76 & 0.60 \\
\hline Sentirse optimista & 0.25 & 0.60 & Sentirse vigoroso & 0.21 & 0.60 \\
\hline Estado de salud & 0.44 & 0.56 & Sentirse estresado & 0.56 & 0.56 \\
\hline Sentirse estresado & 0.57 & 0.56 & Estado de salud & 0.43 & 0.55 \\
\hline $\begin{array}{c}\text { Índice de Exclusión } \\
\text { Social }\end{array}$ & 0.79 & 0.55 & Sentirse solo & 0.52 & 0.54 \\
\hline Sentirse solo & 0.52 & 0.54 & Índice de Exclusión Social & 0.80 & 0.52 \\
\hline $\begin{array}{c}\text { Calidad de los } \\
\text { servicios sanitarios }\end{array}$ & 0.92 & 0.47 & $\begin{array}{c}\text { Calidad de los servicios } \\
\text { sanitarios }\end{array}$ & 0.92 & 0.46 \\
\hline Mortalidad Infantil & 0.94 & 0.39 & Mortalidad Infantil & 0.91 & 0.44 \\
\hline Esperanza de vida & 0.80 & 0.33 & Esperanza de vida & 0.73 & 0.36 \\
\hline $\begin{array}{c}\text { Esperanza de vida } \\
\text { saludable }\end{array}$ & 0.84 & 0.30 & $\begin{array}{c}\text { Esperanza de vida } \\
\text { saludable }\end{array}$ & 0.82 & 0.30 \\
\hline
\end{tabular}

Así, se puede observar que en ambos casos la estructura del ISS es similar, puesto que el índice de salud mental es el indicador simple más correlacionado con el ISS e incorpora toda su información. Este hecho puede deberse a que la salud mental, aparte de ser uno de los indicadores objetivos incluidos en el ISS, es un indicador muy importante a la hora de explicar factores como la longevidad y los comportamientos relacionados con la salud (Pressman y Cohen, 2006) o la accesibilidad al mercado laboral (Evans-Lacko et al., 2013). En este último caso, son las personas desempleadas las que tienden a tener una salud mental 
más pobre, presentando, además, mayores probabilidades de exclusión social y de estar en riesgo de pobreza (Barr et al., 2015). Los siguientes indicadores parciales en acceder a ambos ISS son aquellos relacionados con sentirse alegre, deprimido y relajado, que incorporan aproximadamente un 30\%, 70\% y 30\% respectivamente de su información al ISS, debido a que el porcentaje restante es información ya contenida en los indicadores parciales precedentes.

Hay que destacar que el indicador simple de estado de salud, normalmente empleado como un indicador global para medir la salud, accede al Indicador Sintético en el octavo puesto en el caso de los empleados, y en noveno puesto en el caso de los desempleados. Este hecho puede sugerir que los individuos, a la hora de valorar su salud, se ven influenciados por su estado anímico en el momento de la encuesta (Manderbaka, 1998).

Los últimos indicadores que acceden a los ISS son la mortalidad infantil, la esperanza de vida y la esperanza de vida saludable, lo que implica que están menos correlacionados con el Indicador Sintético, aunque aportan un gran porcentaje de información nueva. Esto puede deberse a que se trata de indicadores a nivel macro, es decir, que adquieren el mismo valor por país, por lo que su grado de dispersión es más reducido. No obstante, estos indicadores son de gran importancia para medir los cambios en el estado de salud de una población, ya que existen desigualdades en los niveles de mortalidad entre países y entre grupos socioeconómicos dentro de cada uno de los países (Robine, Romieu y Camboir, 1999). Por ejemplo, la esperanza de vida de las mujeres es superior a la de los hombres, sin embargo, la esperanza de vida saludable, es decir, la proporción de vida sin enfermedad, es ligeramente inferior en el caso de las mujeres (Mathers et al., 2001). Así, se ha demostrado que la esperanza de vida saludable es un buen indicador para medir la salud de una población, puesto que tiene en cuenta los cambios en su estado físico y mental (Robine y Ritchie, 1991). Por otra parte, aunque el indicador de mortalidad infantil ha recibido críticas por ser una medida centrada en una pequeña parte de la población, Reidpath y Allotey (2003) demostraron que se trata de un indicador sensible a cambios estructurales como el desarrollo económico, las condiciones de vida en general, el bienestar social, y la calidad del entorno que, consecuentemente, afectan a toda la población en general. Para ello, relacionaron el indicador de mortalidad infantil con la esperanza de vida ajustada por incapacidad, conocida por sus siglas en inglés como DALE (Disability Adjusted Life Expectancy), en 180 países y encontraron una relación lineal y altamente significativa entre ambos indicadores. Por todo ello, aunque los indicadores a nivel macro estén menos correlacionados con el ISS, se trata de indicadores de gran importancia para medir el nivel general de salud de una población amplia y diversa como es el caso de la Unión Europea. 


\subsection{Análisis comparativo de los indicadores sintéticos de salud por situación laboral}

Con el fin de comparar los ISS obtenidos para empleados y desempleados y comprobar su comportamiento a lo largo de los 28 países que conforman la Unión Europea, se han tipificado previamente los valores de ambas distribuciones, de manera que valores negativos indican que nos encontramos por debajo de la media de cada una de las distribuciones y valores positivos por encima.

La Figura 1 muestra que la salud de los empleados y los desempleados es mayor en los países del norte, centro y sur de Europa, mientras que en los países del este se muestran valores más pobres del ISS tanto de empleados como de desempleados. Si atendemos a las diferencias existentes en la salud de empleados y desempleados, podemos ver que entre los países que están por encima de la media (valores positivos), la salud de los empleados es mejor que la de los desempleados en la mayoría de los casos, no obstante, las diferencias no son muy acusadas. Este resultado no es sorprendente, puesto que se trata precisamente de aquellos países en los que sus respectivos sistemas de bienestar están más desarrollados, protegiendo así a ambos colectivos.

Por el contrario, entre los países que están por debajo de la media, la salud de los empleados fue más pobre que la de los desempleados, con grandes diferencias en el caso de Letonia y Rumania. En este caso entran en juego las condiciones laborales a las que están expuestos los empleados que desarrollan su trabajo en estos países, que tienden a ser más pobres y afectar, por lo tanto, negativamente a su salud. 


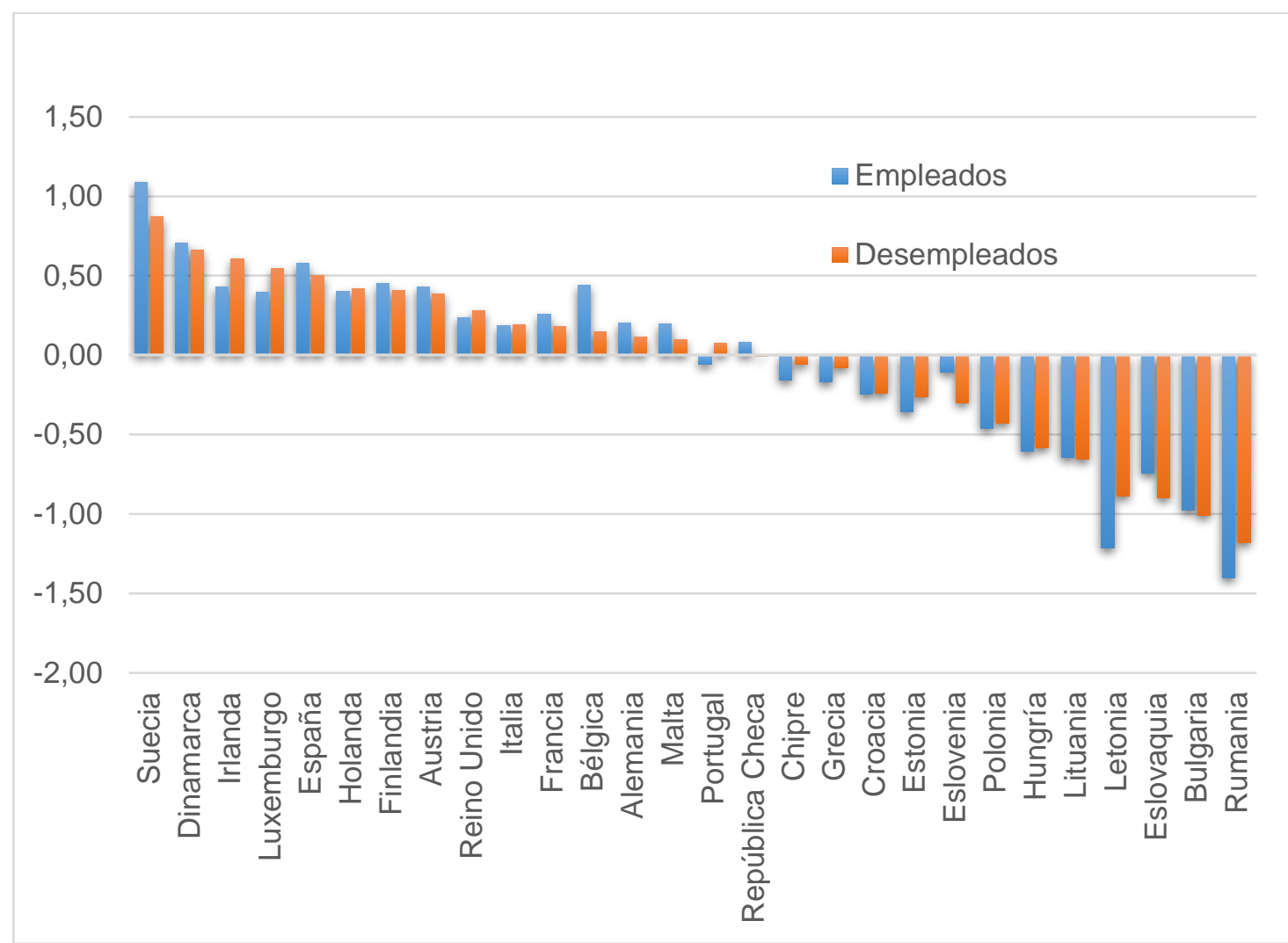

Figura 1. Indicadores Sintéticos de Salud de empleados y desempleados por países

\subsection{Análisis de los indicadores sintéticos de salud por género}

Uno de los objetivos del presente trabajo de investigación es comprobar la existencia de diferencias de género. Para ello, hemos calculado, por un lado, las diferencias entre empleados y desempleados en función de su sexo y, por otro, las existentes entre hombres y mujeres en función de su situación laboral. Las diferencias se obtienen a partir de restar los valores de ambas distribuciones.

La Figura 2 refleja el primer caso, es decir, las diferencias entre empleados y desempleados en función de su sexo. Hay que tener en cuenta que el gráfico muestra las diferencias entre empleados y desempleados del mismo sexo, por lo que valores positivos indican mejor salud para los empleados y valores negativos lo indican para los desempleados.

Los países donde la salud de los empleados y las empleadas fue mejor que la de las personas en situación de desempleo fueron Alemania, Bélgica, Eslovaquia, Eslovenia, Finlandia, España, Repúbica Checa, Suecia y Dinamarca. Por el contrario, la salud de los desempleados fue mejor que la de las personas en situación de empleo tanto para los hombres como para las mujeres en Estonia, Grecia, Holanda, Irlanda, Letonia, Luxemburgo, Portugal, Reino Unido y Rumania. No obstante, en países como Austria, Croacia, Francia, Hungría, Bulgaria y Malta, los hombres obtienen mejores resultados en el ISS cuando están 
desempleados y las mujeres cuando están empleadas. Finalmente, en Italia, Lituania, Polonia y Chipre sucede lo contrario, el ISS de los hombres es mayor cuando están empleados y el ISS de las mujeres cuando están desempleadas. Este hecho sugiere que no existe un patrón territorial claro en cuanto a las diferencias en salud entre empleados y desempleados cuando se considera su sexo.

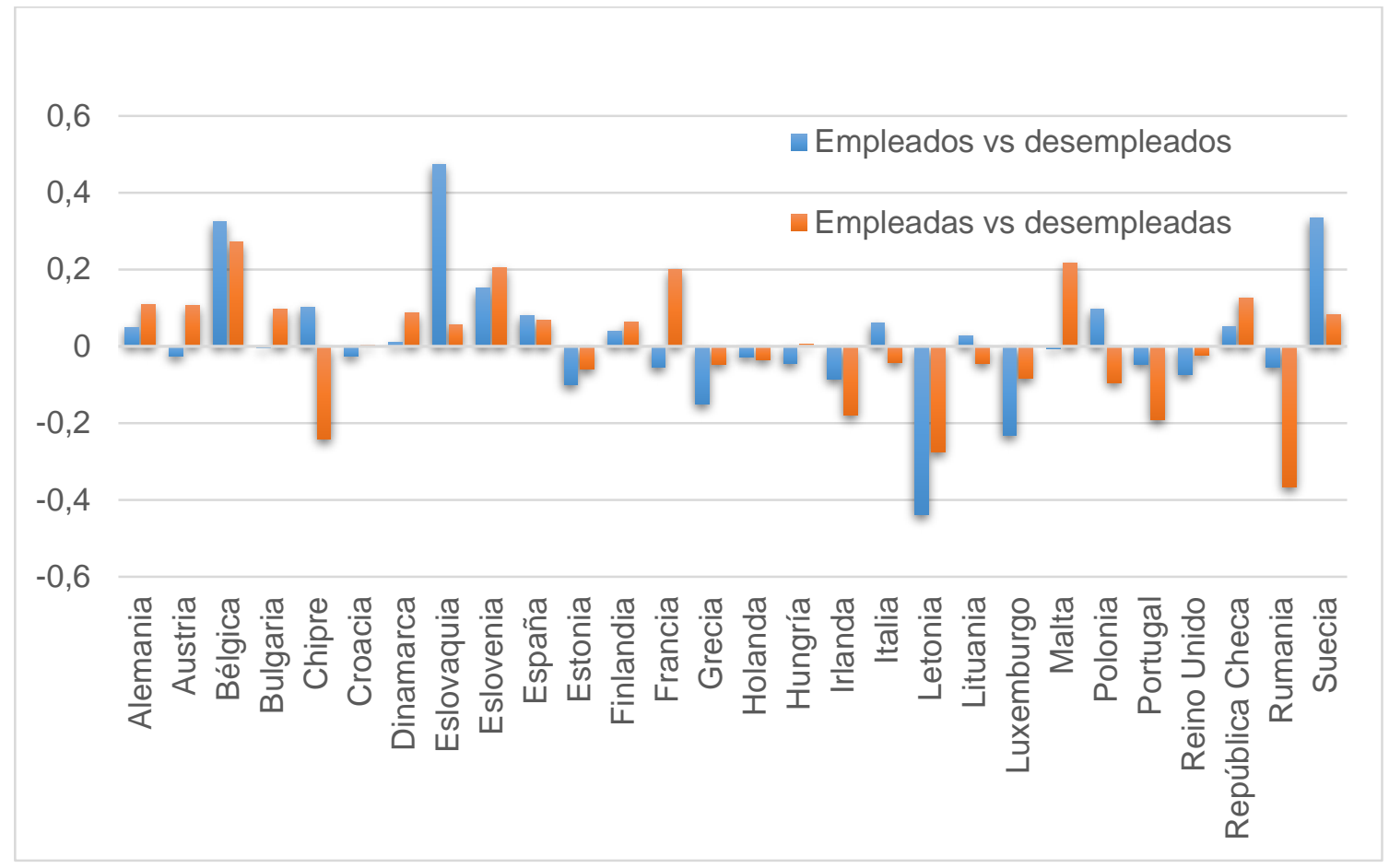

Figura 2. Diferencias entre empleados y desempleados por sexo

Por otra parte, también se han calculado las diferencias en salud entre hombres y mujeres en función de su situación laboral (Figura 3). Los valores positivos indican mejor salud para los hombres y los negativos lo indican para las mujeres. Se puede comprobar que en 18 de los 28 países analizados, la salud de las mujeres es mejor que la de los hombres, especialmente cuando se encuentran en situación de empleo. No obstante, en siete de esos países, Bélgica, Eslovaquia, España, Irlanda, Lituania, Polonia y Suecia, la salud de las mujeres es mejor cuando se encuentran desempleadas. Este último resultado podría deberse a que las mujeres, al encontrarse desempleadas, tienen más tiempo para dedicarlo a la familia y otros aspectos de la vida (Virtanen et al., 2003), lo que puede repercutir positivamente en su salud.

La salud de los hombres, tanto empleados como desempleados, fue mejor que la de las mujeres en su misma condición laboral únicamente en cinco países: Chipre, Dinamarca, Holanda, Portugal y Reino Unido. Finalmente, en algunos países la salud de hombres y mujeres difiere según su situación laboral. Así, en Austria, Francia y Malta, la salud de las mujeres es mejor cuando están empleadas y la de los hombres cuando están desempleados. 
En cambio, en Italia y Rumania sucede lo contrario, la salud de las mujeres es mejor cuando están desempleadas y la de los hombres cuando se encuentran en situación de empleo.

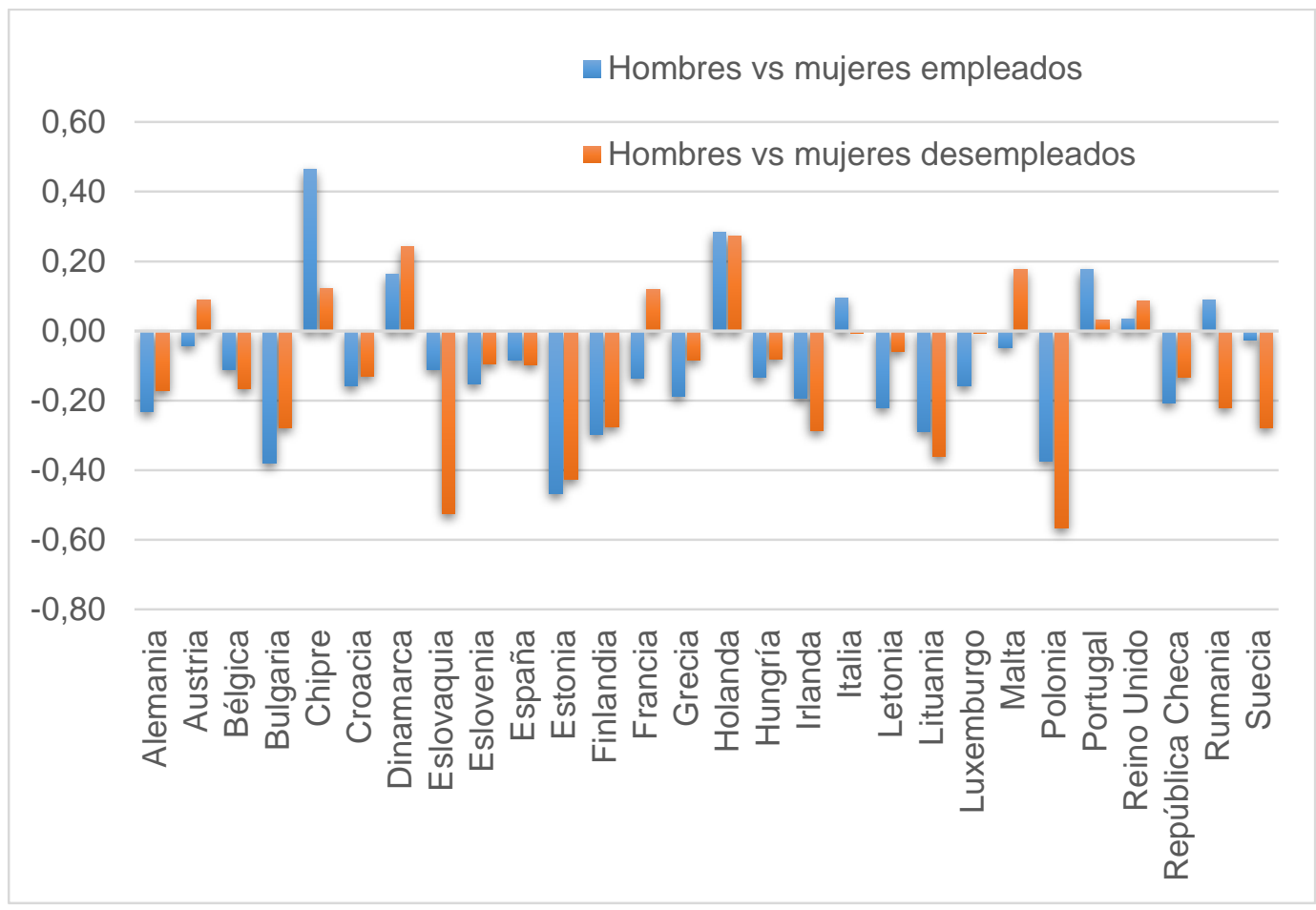

Figura 3. Diferencias entre hombres y mujeres por situación laboral

\section{Discusión}

Los Indicadores Sintéticos de Salud muestran un patrón territorial a lo largo de la Unión Europea caracterizado por un dualismo entre los países del norte, centro y sur de Europa, y los países de la Europa del este. Entre estos últimos, la salud de los empleados ha resultado ser más pobre que la de las personas en situación de desempleo. Este hecho también se da en varios países del norte, centro y sur de Europa, aunque las diferencias no son muy acusadas entre ambos colectivos.

En este sentido, la hipótesis inicial de que el trabajo repercute positivamente en la salud no se cumpliría en la mayoría de los países de la Unión. Aunque estos resultados pueden resultar contradictorios con trabajos previos que demuestran que la salud de los desempleados es más pobre que la de las personas con un trabajo permanente (Urbanos Garrido y López Valcárcel, 2013 y Minelli et al., 2014), hay que tener en cuenta que este estudio aborda las desigualdades en salud con un Indicador Sintético que incluye diversos indicadores relacionados con la salud, las emociones y el estado anímico en el momento de la encuesta. Este hecho, unido a que las personas desempleadas tienen más tiempo que las empleadas para realizar hábitos de tipo saludable como ir al gimnasio o salir a correr, añadido 
a no verse afectadas por las situaciones de estrés que genera el trabajo, puede explicar parcialmente los resultados.

Por otro lado, debido a limitaciones en nuestra base de datos, no hemos tenido en cuenta el tipo de trabajo remunerado que poseen las personas empleadas, ya que en función de si el trabajo es precario o no, la salud de los individuos se ve claramente afectada. Kim et al. (2008), así como Vives et al. (2013), demostraron que las personas que sufren precariedad laboral reportan una peor salud, y que esta condición es más común entre las mujeres.

Nuestros resultados sugieren que la salud de las mujeres empleadas es mejor que la de los hombres en su misma condición laboral a pesar de que tienen más riesgo de estar contratadas en trabajos precarios. Sin embargo, como se ha reflejado anteriormente, los ISS que hemos construido tienen en cuenta otras variables además de la salud, como son las emociones. Nuestra muestra indicaba que las mujeres reportan unos sentimientos de optimismo, relajación y vigorosidad mayores que los hombres, lo que puede explicar en cierta medida los resultados. Este hecho, unido al empleo de un mayor número de indicadores subjetivos que objetivos, puede influenciar la obtención de dicho resultado. Además, si tenemos en cuenta que los trabajos atípicos implican menos horas de trabajo y, consecuentemente, más horas para dedicar a la familia y otros aspectos de la vida, los resultados positivos del ISS para las mujeres se pueden ver justificados.

En definitiva, este trabajo de investigación, a pesar de las limitaciones expuestas anteriormente, determina que la situación laboral de los individuos afecta a la salud de manera diferenciada de hombres y mujeres, y con dependencia del país de residencia. Por lo tanto, en este sentido, entran en juego las políticas que cada uno de los países adopten en materia laboral y familiar, ya que podrían ayudar a paliar las desigualdades existentes en salud entre los hombres y las mujeres. Estos aspectos, junto a las condiciones y tipo de trabajo, supondrían un tema interesante para futuras investigaciones, ya que ayudarían a arrojar más luz sobre las desigualdades en salud existentes entre los hombres y las mujeres en el mercado laboral.

\section{Referencias bibliográficas}

Artazcoz, L., Cortes, I., Puig-Barrachina, V., Benavides, F.G., Escriba-Aguir, V., y Borrell, C. (2014). Combining employment and family in Europe: the role of family policies in health. Eur J Public Health, 24(4), 649-55.

Barr, B., Kinderman, P. y Whitehead, M. (2015). Trends in mental health inequalities in England during a period of recession, austerity and welfare reform 2004 to 2013. Soc Sci Med, 147, 324-31. 
Böckerman, P. y llmakunnas, P. (2009). Unemployment and self-assessed health: evidence from panel data. Health Economics, 18(2), 161-179. doi: 10.1002/hec.1361

Chen, M., Bush, J.W., Patrick, D. (1975). Social indicators for health planning and policy analysis. Policy Sci, 6(1), 71-89.

Evans-Lacko, S., Knapp, M., McCrone, P., Thornicroft, G. y Mojtabai, R. (2013). The Mental Health Consequences of the Recession: Economic Hardship and Employment of People with Mental Health Problems in 27 European Countries. PLoS One, 8(7), e69792.

Kawachi, I. (2008). Globalization and workers' health. Industrial health, 46(5), 421-423.

Kim, M. H., Kim, C. Y., Park, J. K., y Kawachi, I. (2008). Is precarious employment damaging to self-rated health? Results of propensity score matching methods, using longitudinal data in South Korea. Soc Sci Med, 67(12), 1982-1994.

Klomp, J. y de Haan, J. (2010). Measuring Health: A Multivariate Approach. Soc Indic Res, 96(3), 433-457.

Manderbacka, K. (1998). Examining what self-rated health question is understood to mean by respondents. Scand J Public Health, 26(2), 145-153.

Mathers, C.D., Sadana, R., Salomon, J.A., Murray, C.J. y Lopez, A.D. (2001). Healthy life expectancy in 191 countries, 1999. Lancet, 357(9269),1685-1691

Menéndez, M., Benach, J., Muntaner, C., Amable, M. y O'Campo, P. (2007). Is precarious employment more damaging to women's health than men's? Soc Sci Med, 64(4), 776-781.

Michalos, A.C. (2003). Social Indicators Research and Health-Related Quality of Life Research. En A.C., Michalos (Ed.), Essays on the Quality of Life. (pp. 239-271). Netherlands: Springer Netherlands.

Minelli, L., Pigini, C., Chiavarini, M. y Bartolucci, F.(2014). Employment status and perceived health condition: Iongitudinal data from Italy. BMC public health., 14(1), 946. doi: 10.1186/1471-2458-14-946.

OMS. (2011). Gender, health and work. Geneva, Switzerland: World Health Organization.

OMS. (2016). Women's health and well-being in Europe: beyond the mortality advantage. Copenhagen, Denmark: World Health Organization.

Paul, K. I., y Moser, K. (2009). Unemployment impairs mental health: Meta-analyses. Journal of Vocational Behavior, 74(3), 264-282.

Pressman, S.D., y Cohen, S. (2005). Does Positive Affect Influence Health? Psychol 
Bull,131(6), 925-971.

Quesnel-Vallée, A., DeHaney, S. y Ciampi, A. (2010). Temporary work and depressive symptoms: A propensity score analysis. Soc Sci Med, 70(12), 1982-1987.

Reidpath, D.D. y Allotey, P. (2000). Infant mortality rate as an indicator of population health. $J$ Epidemiol Community Heal, 57, 344-346.

Robine, J.M., Romieu, I. y Cambois, E. (1999). Health expectancy indicators, Bulletin of the World Health Organization, 77(2), 181-185.

Robine, J.M. y Ritchie, K. (1991). Healthy life expectancy: evaluation of global indicator of change in population health. BMJ, 302(6774), 457-460.

Rugulies, R., Aust, B., Burr, H., y Bültmann, U. (2008). Job insecurity, chances on the labour market and decline in self-rated health in a representative sample of the Danish workforce. Journal of Epidemiology and Community Health, 62(3), 245-250.

Somarriba, N., Pena, B. (2009). Synthetic indicators of quality of life in Europe. Soc Indic Res, 94(1), 115-133. doi:10.1007/s11205-008-9356-y.

Somarriba, N., Zarzosa, P. (2016). Quality of Life in Latin America: A Proposal for a Synthetic Indicator. En G., Tonon (Ed.), Indicators of Quality of Life in Latin America. (pp. 1956). Cham: Springer International.

Urbanos-Garrido, R. M. y Lopez-Valcarcel, B. G. (2013). Desempleo y salud: Un análisis de la repercusión de la crisis económica sobre la salud de los españoles. Estudios de Economía Aplicada, 31(2), 303-326.

Virtanen, P., Liukkonen, V., Vahtera, J., Kivimäki, M. y Koskenvuo, M. (2003). Health inequalities in the workforce: the labour market core-periphery structure. International Journal of Epidemiology, 32(6), 1015-1021.

Vives, A., Amable, M., Ferrer, M., Moncada, S., Llorens, C., Muntaner, C., Benavides, F.G. y Benach, J. (2013). Employment precariousness and poor mental health: evidence from Spain on a new social determinant of health. Journal of Environmental and Public Health, 2013(2013), 978656. doi: 10.1155/2013/978656

Zarzosa Espina, P. (1996). Aproximación a la medición del bienestar social. Idoneidad del indicador sintético "Distancia-P2". (Aplicación al caso español). Cuad Econ, $24,139-163$. 